\title{
SHEARING TESTS APPLIED TO PANTOGRAPHIC STRUCTURES
}

\author{
Gregor Ganzosch ${ }^{a, *}$, Francesco dell'Isola ${ }^{b}$, Emilio Turco $^{c}$, \\ TOMASZ LEKSZYCKI ${ }^{d}$, WOLFGANG H. MÜLleR ${ }^{a}$ \\ ${ }^{a}$ TU Berlin, Chair of Continuum Mechanics and Materials Theory, Einsteinufer 5, Berlin, Germany \\ ${ }^{b}$ La Sapienza University, Dipartimento di Ingegneria Strutturale e Geotecnica, Via Eudossiana 18, Rome, Italy \\ ${ }^{c}$ UNISS, Dipartimento di Architettura, Design e Urabanistica, via Garibaldi 35, Alghero, Italy \\ d PAN Warszawa, Instytut Podstawowych Problemów Techniki, ul. Pawinskiego 5B, Warszawa, Poland \\ * corresponding author: ganzosch@tu-berlin.de
}

\begin{abstract}
.
With the advancements in 3D printing technology, rapid manufacturing of fabric materials with complex geometries became possible. By exploiting this technique, different materials with different structures have been developed in the recent past with the objective of making generalized continuum theories useful for technological applications. So-called pantographic structures are introduced: Inextensible fibers are printed in two arrays orthogonal to each other in parallel planes. These superimposed planes are inter-connected by elastic cylinders. Five differently-sized samples were subjected to shear-like loading while their deformation response was analyzed. Results show that deformation behavior is strong non-linear for all samples. Furthermore, all samples were capable to resist considerable external shear loads without leading to complete failure of the whole structure. This extraordinary behavior makes these structures attractive to serve as an extremely tough metamaterial.
\end{abstract}

KEYWORDs: Pantograpghic structures, shearing tests, generalized continuum theories.

\section{INTRODUCTION}

Micro-Electro-Mechanical-Systems (MEMS) are rapidly becoming smaller. The miniaturization of these systems requires new constitutive equations for describing their mechanical and material behavior (e.g., the deformation pattern of accelerometer sensors in cars). In [1] it was shown that the elastic modulus of epoxy increases if the outer dimensions of the specimen become smaller. The reason for this behavior is hidden in an internal substructure. Such a phenomenon is a.k.a. size effect and it characterizes the departure of mechanical behavior from classical continuum mechanics when changing the outer dimension. However, this effect is not limited to the microscopic world. In the literature size effects of different materials of all length scales can be found, starting from investigations on block masonry in the meter-scale [17], continuing with experiments on polymer foams in the millimeter range [10, on to studies on the micrometer-scale in silicon beams [5], and ending finally with bending experiments of nanowires on the nanometer-scale [18].

Another example of intrinsic length scales in complex macroscopic specimens are so-called pantographic structures (see Fig. 1), which were developed and intensively studied by dell'Isola and his team in [2]. Here the length and elastic properties of the constituting trusses in combination with the stiffness of their joints form the internal platform on which their macroscopic deformation behavior relies. Such intrinsic length scale parameters are the true reason behind what is phenomenologically called size effect. Classical elasticity theory of continuum mechanics is not able to explain this kind of material behavior because it does not contain an intrinsic length scale. This is why generalized continuum theories must be used to overcome such limits. New material parameters show up during the development of the corresponding constitutive equations. Therefore experiments have to be performed, by means of which these new and unknown parameters can be determined.

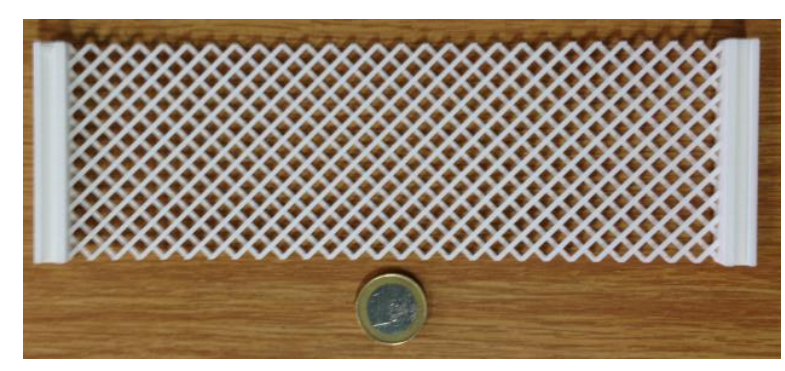

FIgURE 1. Example of a pantographic structure developed by 2 .

In recent years, accompanied by the advancements in 3D printing technology, new experimental techniques have been developed with the objective of making generalized continuum theories useful in technology [1-3, 5, 8]. Different experimental set-ups (microscopic as well as macroscopic) when using different materials with complex structures (e.g., static and dynamic bending tests on foams) were developed [1, 2, 5, 8, 10, 14. An experiment was performed on 
so-called pantographic structures by 2]. Two families of inextensible fibers, which can also be treated as beams, are 3D-printed in two arrays orthogonal to each other in parallel planes. These are then superimposed. The parallel planes are inter-connected by cylindrical elastic pivots of different diameters and heights. Based on a second gradient continuum model [2, 4, 11, 15], the deformation behavior of the samples was assessed in numerical simulations during an extension test. Non-linear models with specific intrinsic parameters led to non-classical behavior in the simulation 2. Based on these results, real macroscopic tensile tests were designed and performed, resulting in an elongation along the direction of the shorter sides of the pantographic samples:

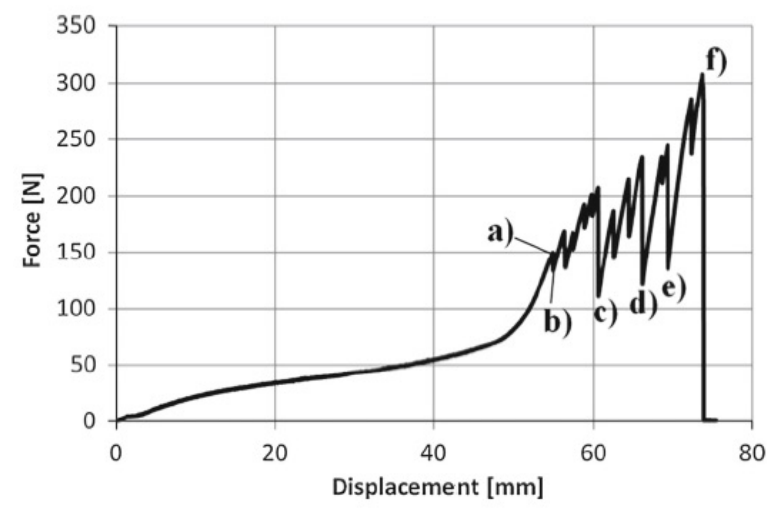

Figure 2. Results of a tensile test applied to a pantographic structure with a pivot height of $0.5 \mathrm{~mm}$ and a pivot diameter of $0.9 \mathrm{~mm}$ from [2].

Force over displacement is plotted in Fig. 2. The failure of the pantographic lattice is visualized by local minima, marked by b), c), d) and e) in Fig. 2 Each minimum relates to fracture of a beam or a pivot. Surprisingly after one failure within the structure, which was subjected to external loading, the specimen recovers and is able to carry even higher loads than before. The objective of this work is to find out in experiments as to whether the pantographic structures show the same non-linear behavior in shearing tests.

\section{Material And Methods}

Because of their complex periodic structure, the pantographic samples considered for the investigations in shearing tests were 3D-printed. Polyamide powder was used as raw material. 3D-models were generated by using a commercial CAD software and saving them in STL format file, which was then used as input for the $3 \mathrm{D}$ printer. Five variations of specimens with different inner geometries but equal material densities were investigated. The pantographic structure consists of rectangular beams and cylindrical pivots. A schematic shows different beam and pivot parameters in Fig. 3. Details can be found in Tab. 1. The inner parameters of the pivots are kept constant (except for the diameter of sample $\mathrm{E}$ with $d=3.00 \mathrm{~mm}$, see

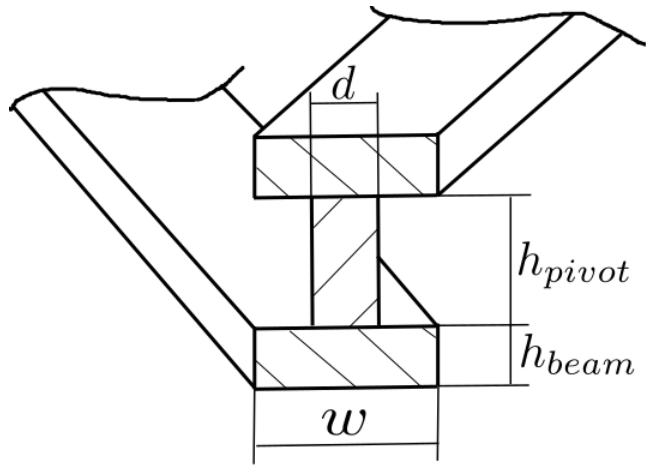

FiguRE 3. Schematic cross-section of a periodic cell of a pantographic structure.

\begin{tabular}{c|c|c|c|c} 
Sample & $w$ & $h_{\text {beam }}$ & $d$ & $h_{\text {pivot }}$ \\
\hline A & $1.00 \mathrm{~mm}$ & $1.00 \mathrm{~mm}$ & $0.90 \mathrm{~mm}$ & $1.00 \mathrm{~mm}$ \\
$\mathrm{~B}$ & $1.60 \mathrm{~mm}$ & $1.00 \mathrm{~mm}$ & $0.90 \mathrm{~mm}$ & $1.00 \mathrm{~mm}$ \\
$\mathrm{C}$ & $1.60 \mathrm{~mm}$ & $1.60 \mathrm{~mm}$ & $0.90 \mathrm{~mm}$ & $1.00 \mathrm{~mm}$ \\
$\mathrm{D}$ & $2.25 \mathrm{~mm}$ & $1.60 \mathrm{~mm}$ & $0.90 \mathrm{~mm}$ & $1.00 \mathrm{~mm}$ \\
$\mathrm{E}$ & $2.25 \mathrm{~mm}$ & $1.60 \mathrm{~mm}$ & $0.90 \mathrm{~mm}$ & $3.00 \mathrm{~mm}$
\end{tabular}

TABLE 1. Overview of five specimens with different beam and pivot parameters.

Tab. 1), so that investigations of pantographic structures will focus on different beam parameters and their influence on the deformation behavior during shear tests. The outer dimension of every specimen is kept constant (length $=204 \mathrm{~mm}$, width $=70 \mathrm{~mm}$ ).

An overview of the whole experimental setup used for the shear tests on planar pantographic sheets is shown in Fig. 4

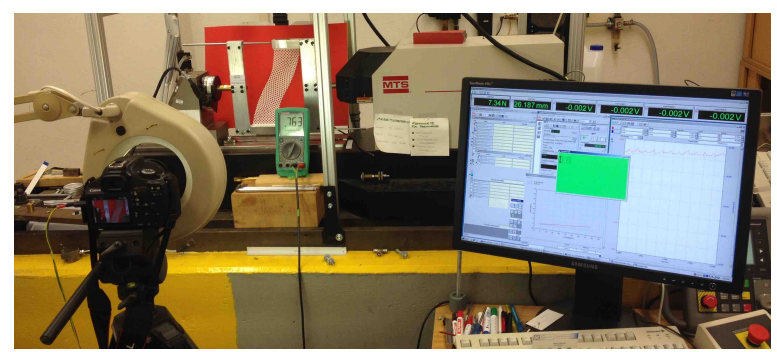

FiguRE 4. Experimental setup during shear deformation: Camera and illumination devices on the left, pantographic sample mounted in an MTS Tytron 250 testing machine in the middle, interface of control software on the right.

An MTS Tytron 250 testing-device controlled by the software Stationsmanager V 3.14 was used during the shearing tests. Applied force was measured by a load cell attached to the device, which is able to record axial forces in a range of $N= \pm 250 \mathrm{~N}$. The displacement was imposed horizontally on the top of the specimen with a loading rate of $v=15 \mathrm{~mm} / \mathrm{min}$. The experiments were displacement-controlled using a DC-linear motor in combination with a spindle. Displacement was measured and monitored by a deviceown encoder unit. Almost frictionless movement was 


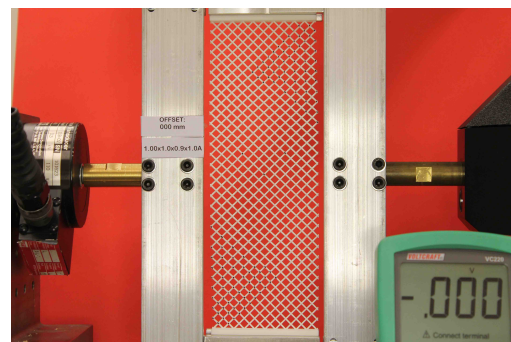

(A) . Mounted sample A before deformation.

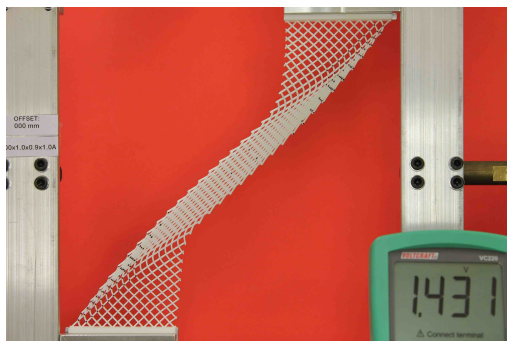

(в) . Deformed sample A directly before first failure occurs.

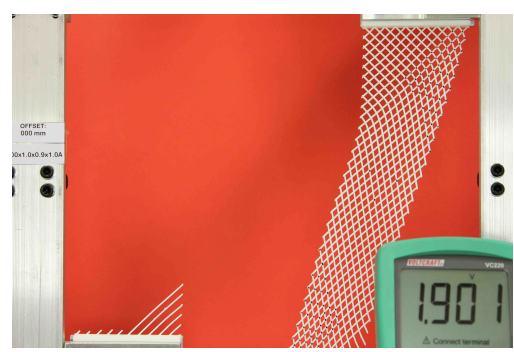

(c) . Sample A after complete failure.

Figure 5. Shear deformation of sample A.

achieved by using an air-film-bearing. External vibration was avoided by using a massive substructure and by arranging the system horizontally. Additionally to force-displacement recording, pictures were taken ( 0.25 pictures/second) by means of a commercial Canon EOS 1000D camera with a resolution of $4272 \times 2848$ pixels. Free software ImageJ 1.50i was used to evaluate image data. Open source software SciDAVis 1.14 was used for preparation, manipulation, and evaluation of acquired machine data.

Fig. 5 shows the deformation of sample A before, during, and after the shearing test.

\section{Results}

Results of all five samples are visualized in Fig. 6 with the help of a force-displacement diagram. Local minima in Fig. 6 6 show a failure of a beam or a pivot. It can be recognized that all samples show different and pronounced non-linear behavior. In order to visualize the results for a discussion, the graph of each sample is plotted separately, starting with sample A in Fig. 7 and ending with sample E in Fig. 11 .

In sample A the first failure occurs at $F_{A}=26.0 \mathrm{~N}$.

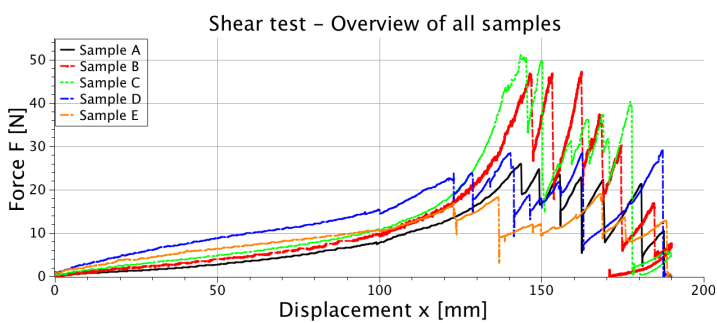

Figure 6. Raw-data of all samples; force $F$ is plotted over displacement $x$.

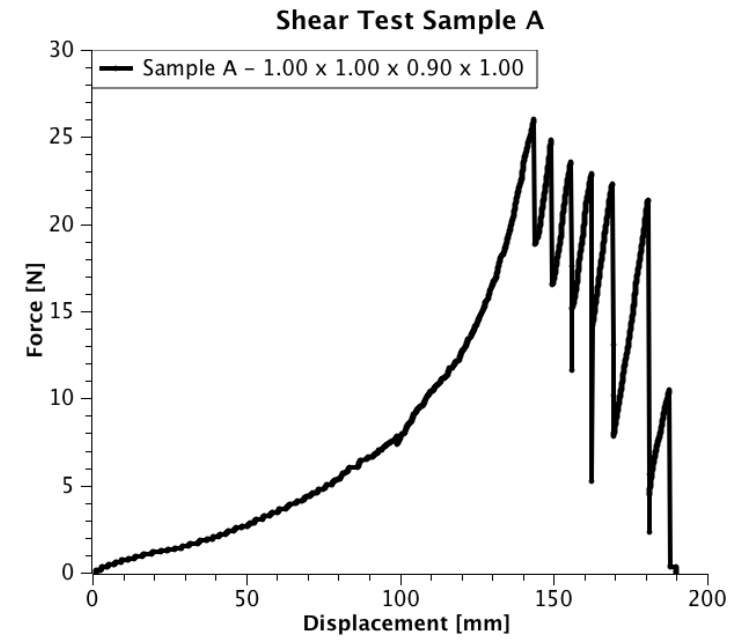

Figure 7. Raw-data of sample A; force $F$ is plotted over displacement $x$.

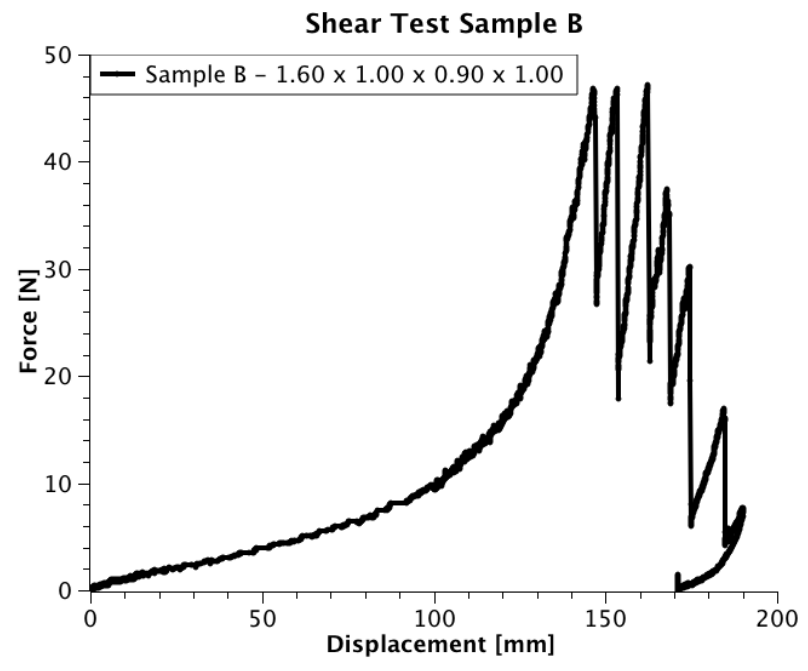

Figure 8. Raw-data of sample B; force $F$ is plotted over displacement $x$.

Surprisingly, samples B $\left(F_{B}=46.9 \mathrm{~N}\right)$ and $\mathrm{C}\left(F_{C}=\right.$ $50.5 \mathrm{~N})$ are able to resist the highest loads. Sample D resists until $F_{D}=23.9 \mathrm{~N}$. Sample E shows the lowest strength and failure occurs at about $F_{E}=16.8 \mathrm{~N}$. In sample $\mathrm{A}$ and $\mathrm{B}$, a beam failure occurs on the lower left corner (shown for sample A in Fig. 12). In contrast to the lower right corner, where just low deformation occurs, the beams at the lower left corner experience a large elongation between the pivots. In 


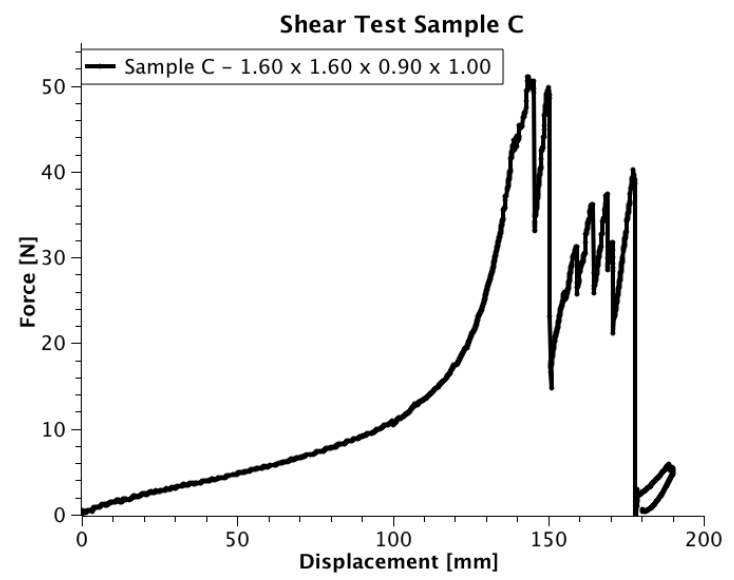

Figure 9. Raw-data of sample C; force $F$ is plotted over displacement $x$.

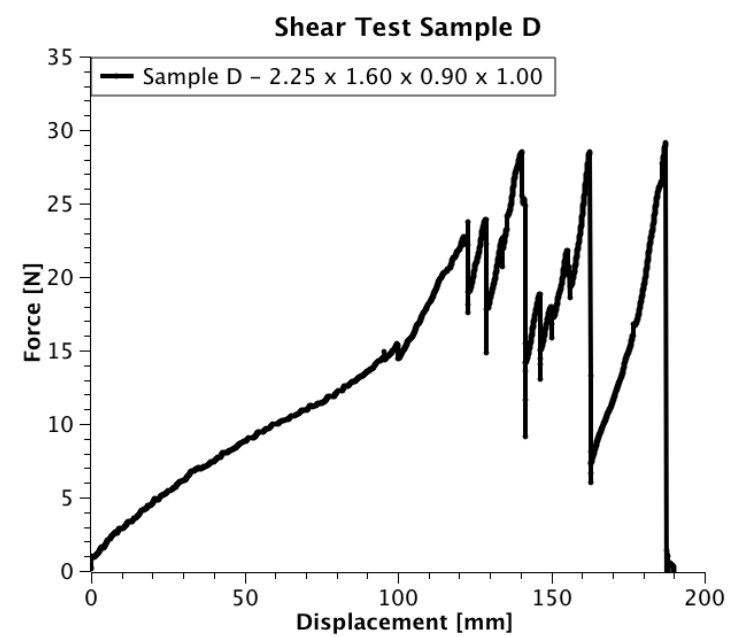

Figure 10. Raw-data of sample D; force $F$ is plotted over displacement $x$.

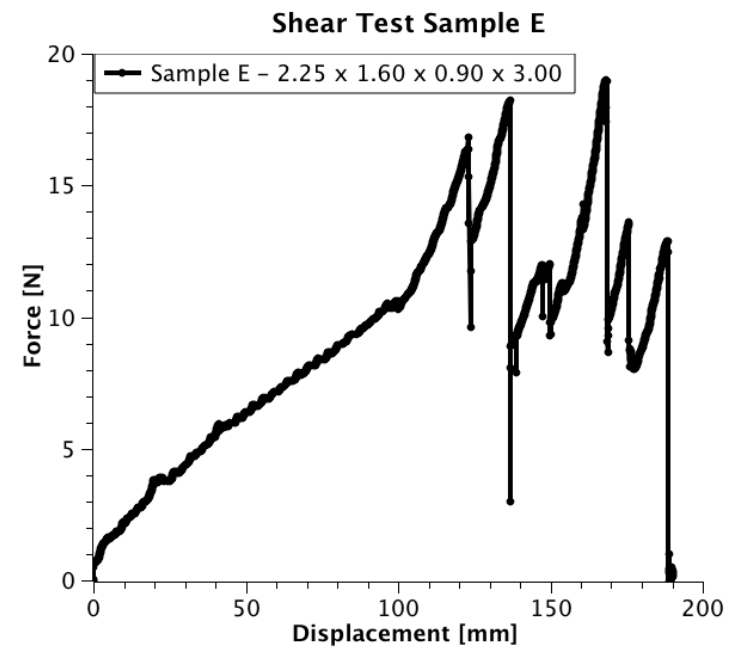

FiguRE 11. Raw-data of sample E; force $F$ is plotted over displacement $x$.

this region, bending energy of fibers is concentrated leading to failure of a beam. First failure of sample $\mathrm{C}, \mathrm{D}$, and $\mathrm{E}$ is quite different (shown for sample $\mathrm{C}$ in
Fig. 13). Obviously, bending energy of fibers is so high in the middle-left that pivots in this region (marked by the circle in Fig. 13p start to separate from the beam structure. Notice, that the variation of inner parameters leads to different deformation and rupture behavior. The influence of inner parameters on the material behavior of the specimen will be described and discussed.

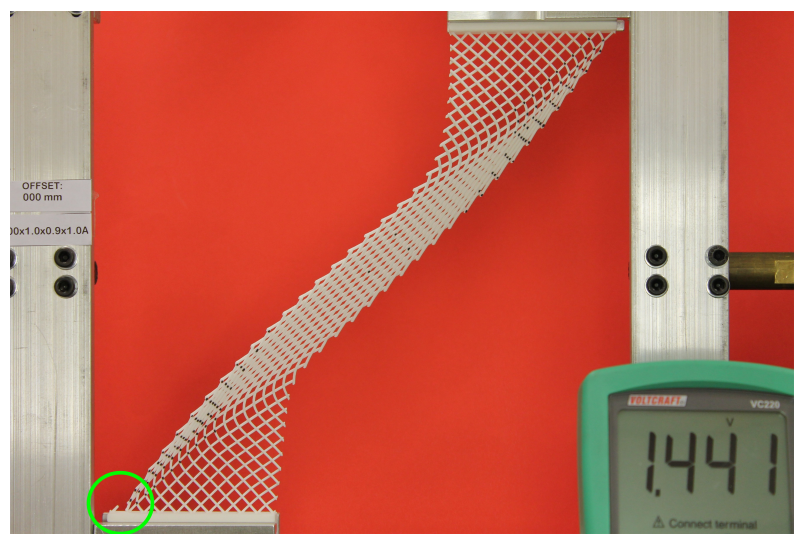

Figure 12. Deformed sample A directly after first failure occurs, region marked by the circle.

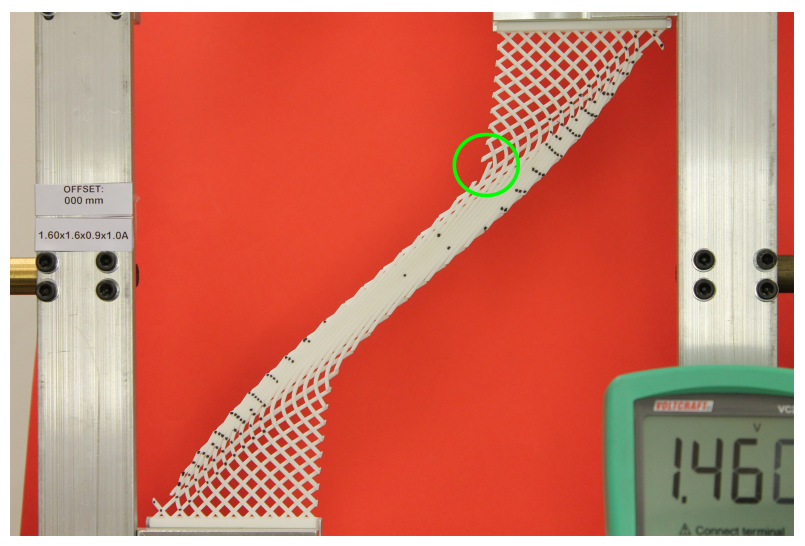

Figure 13. Deformed sample C directly after first failure occurs, region marked by the circle.

By enlarging the width of the beams in the pantographic structure from sample A $(w=1.00 \mathrm{~mm})$ to sample B $(w=1.60 \mathrm{~mm})$, the resistance to shear deformation increases by almost $80 \%$. This enlargement has a very high influence on the strengthening of this structure.

By enlarging the height of the beams from sample B $\left(h_{\text {beam }}=1.00 \mathrm{~mm}\right)$ to sample C $\left(h_{\text {beam }}=1.60 \mathrm{~mm}\right)$, the resistance to shear deformation increases by just $7.7 \%$. This is a much smaller influence compared the one from sample A to B mentioned before. But still sample $\mathrm{C}$ is able to carry the highest shear load until the first failure occurs $\left(F_{C}=50.5 \mathrm{~N}\right)$.

By enlarging the width of the beams from sample C $(w=1.60 \mathrm{~mm})$ to sample $\mathrm{D}(w=2.25 \mathrm{~mm})$, the resistance to shear deformation decreases by $52.7 \%$. 
This observation is contrary to our assumptions and indicates an upper limit value for the width of beams between $1.60 \mathrm{~mm} \leq w<2.25 \mathrm{~mm}$.

Finally, by enlarging the height of the pivots from sample D $\left(h_{\text {pivot }}=1.00 \mathrm{~mm}\right)$ to sample $\mathrm{E}\left(h_{\text {pivot }}=\right.$ $3.00 \mathrm{~mm}$ ), the resistance to shear deformation decreases by $29.7 \%$. This can be explained by the higher lever arm between the beams resulting in a high torque which then leads to failure.

Notice that complete failure for samples A, D, and E occurs at a deformation of about $x=190 \mathrm{~mm}$. Absolute structural failure of the flexible sample B and C could not be investigated due to movement-limitations of machine-axis. This is also the reason for unloading curves in sample B and $\mathrm{C}$, which occurred during the removal procedure of the samples from testing device. Interestingly, sample $\mathrm{A}, \mathrm{B}$, and $\mathrm{C}$ show a strong non-linear increase of their slopes before first failure occurs. This is not the case for sample D and E with higher values for the width of beams $(w=2.25 \mathrm{~mm})$. Furthermore, samples B, D, and E are able to carry even higher loads after first failure. Because of the complex geometry, the beams reorganize themselves resulting in a higher resistance to shear loading. This behavior was also observed during tensile tests for the case of uni-axial loading as afore-mentioned in 2 .

\begin{tabular}{c|c|c|c} 
Sample & $x_{m}[\mathrm{~mm}]$ & $x_{i}[ \pm 1 \mathrm{~mm}]$ & $\triangle x[ \pm 1 \mathrm{~mm}]$ \\
\hline $\mathrm{A}$ & 143.96 & 142.47 & $1.49(1.04 \%)$ \\
$\mathrm{B}$ & 142.91 & 141.50 & $1.41(0.99 \%)$ \\
$\mathrm{C}$ & 145.71 & 145.29 & $0.42(0.29 \%)$ \\
$\mathrm{D}$ & 122.96 & 122.13 & $0.83(0.68 \%)$ \\
$\mathrm{E}$ & 123.77 & 123.49 & $0.28(0.23 \%)$
\end{tabular}

TABLE 2. Listed values of displacement based on image data $\left(x_{i}\right)$ and based on machine data $\left(x_{m}\right)$ at the time when first breakage of the specimen occurred. Difference of displacement between machine data and image data $(\triangle x)$ is presented in the last column.

By comparing image data with machine data, no significant difference in displacement was observed (see Tab. 2). Sample A shows the highest difference between displacement obtained from machine data and main displacement obtained from image data $\left(\triangle x_{A}=1.04 \%\right)$, sample $E$ shows the lowest one $\left(\triangle x_{E}=0.23 \%\right)$. Note that displacements captured by the camera are smaller than the ones obtained from the testing device. We assume, that one reason for this observation is the compliance (inverse of stiffness) of the testing device and its structure: deformation of clamping jaws and mounting support influences the real displacement. Another reason is based on the uncertainty of image data: pictures were taken every 4 seconds (as described in Sect. 2), in which the loading-axis was able to travel up to $1 \mathrm{~mm}$ (error of $\pm 1 \mathrm{~mm}$ in second column of Tab. 2, $x_{i}$ ). This results in a discrepancy between real and measured displacement.

Furthermore it has to be mentioned that image data was just able to capture the deformation behavior in a two dimensional plane. But during the experiments it was observed that the structure was also deforming in the third direction (out-of-plane). This buckling-like behavior varied strongly for each sample. Highest out-of-plane-movements were observed in experiments involving sample A and B (up to $19 \mathrm{~mm}$ ). Sample C, $\mathrm{D}$, and E showed very weak out-of-plane-movements or even none. The investigation of this effect is strongly recommended in further research projects. Manufacturing process (rapid prototyping) may have a big influence on the material behavior as well (e.g., direction of printing or thickness of layer) and should also be part of future investigations. Notice, that each sample was just measured once. To increase the quality of results, each experiment should be repeated under exactly the same experimental conditions. Acquired data should then be compared among each other.

\section{Conclusions}

Deformation behavior of various pantographic structures with different inner parameters was investigated in shearing tests. We showed that the variation of inner parameters (beam and pivot dimensions) have a deep impact on the material behavior. All samples behaved non-linearly and were capable to resist considerable external shear loads without leading to complete failure of the whole structure. Because of the complex geometry beams and pivots reorganize themselves resulting in an higher resistance to outer load, so that even higher loads can be carried after failure (see sample B, D, and E). An aim for further investigations should be to provide a better predictability of structural failure behavior by manipulating the inner parameters in a way that all force-peaks should be at one level. This would make the deformation behavior of the structure more predictable and would contribute to make these structures available for application in technology.

Real experiments are quite involved. Thus, the obtained results shall be used to develop further simulation models as shown in [3] or [14, 15]. It is also possible to investigate the influence of inner parameters on the material behavior in a numerical analysis [14, 16. However, these models are based on many simplifications and restrictions so that a true validation would still be challenging and should be investigated further.

As afore-mentioned in Sect. 3, investigated lattices were just examined in context with two-dimensional planar structures (neglect of twist or buckling). Other forms, such as cylinders or rectangles, would contribute to three-dimensional stability and would help to make these structures useful for technology. In summary one may say that the combination of strength and lightness makes these structures attractive to serve as an extremely tough metamaterial (e.g., airplane frame parts or artificial bone scaffolds). 


\section{LIST OF SYMBOLS}

$F$ Force [N]

$x$ Displacement [mm]

$x_{m}$ Displacement based on machine data [mm]

$x_{i} \quad$ Displacement based on image data $[\mathrm{mm}]$

$\triangle x$ Difference between $x_{m}$ and $x_{i} \quad[\mathrm{~mm}]$

$w$ Width $[\mathrm{mm}]$

$d$ Diameter [mm]

$h_{\text {beam }}$ Beam-height [mm]

$h_{\text {pivot }}$ Pivot-height [mm]

$v$ Velocity $[\mathrm{mm}]$

\section{REFERENCES}

[1] Liebold, C., Müller, W.H. Applications of higher-order continua to size effects in bending: Theory and recent experimental results. Generalized Continua as Models for Classical and Advanced Materials, Springer International Publishing, pp. 237-260, 2016

[2] dell'Isola, F., Lekszycki, T., Pawlikowski, M., Grygoruk, R., Greco, L. Designing a light fabric metamaterial being highly macroscopically tough under directional extension: First experimental evidence. Zeitschrift für angewandte Mathematik und Physik, ZAMP, 66(6), pp. 3473-3498, 2015

[3] dell'Isola, F.,Della Corte, A., Giorgio, I., Scerrato, D. Pantographic 2D sheets: Discussion of some numerical investigations and potential applications. International Journal of Non-Linear Mechanics, Elsevier, 80, pp. 200-208, 2016

[4] Placidi, L., Andreaus, U., Della Corte, A., Lekszycki, T. Gedanken experiments for the determination of two-dimensional linear second gradient elasticity coefficients. Zeitschrift für angewandte Mathematik und Physik, ZAMP, 66(6), pp. 3699-3725, 2015

[5] Liebold, C. Größeneffekt in der Elastizität. Dissertation, Technische Universität Berlin, Germany, 2015

[6] Liebold, C., Müller, W.H. Comparison of gradient elasticity models for the bending of micromaterials. Computational Materials Science. Elsevier, pp. 52-61, 2016

[7] Liebold, C., Müller, W.H. Measuring material coefficients of higher gradient elasticity by using AFM techniques and Raman-spectroscopy. Generalized continua as models for materials, Springer-Verlag Berlin Heidelberg, pp. 255-271, 2013
[8] Ganzosch, G., Müller, W.H. Experimental techniques applied to generalized continuum theories - A state-of-the-art report. Proceedings of the XLIV Summer School-Conference. 44, pp. 149-161, 2016

[9] Lam, D. C. C., Yang, F., Chong A. C. M., Wang, J., Tong, P. Experiments and theory in strain gradient elasticity. Journal of the Mechanics and Physics of Solids. 51(8), pp. 1477-1508, 2013

[10] Lakes, R., Drugan, W.J. Bending of a Cosserat elastic bar of square cross section: Theory and experiment. Journal of Applied Mechanics, 82(9), pp. 2-8, 2015

[11] dell'Isola, F., dAgostino, M. V., Madeo, A., Boisse, P., Steigmann, D. Minimization of shear energy in two dimensional continua with two orthogonal families of inextensible fibers: the case of standard bias extension test. Journal of Elasticity, 122(2), pp. 131-155, 2016

[12] Pipkin, A.C. Plane Traction Problems for inextensible Networks. Q.J. Mech. Appl. Math., 34(4), pp. 415-429, 1981

[13] Rivlin, R.S. Plane Strain of a Net formed by inextensible Cords. Collected Papers of RS Rivlin, Springer Berlin, pp. 511-534, 1997

[14] Turco, E., dell'Isola, F., Rizzi, N.L., Grygoruk, R., Müller, W.H., Liebold, C. Fiber rupture in sheared planar pantographic sheets: Numerical and experimental evidence. Mechanics Research Communications, Elsevier, 76, pp. 86-90, 2016

[15] Turco, E., dell'Isola, F., Cazzani, A., Rizzi, N.L. Hencky-type discrete model for pantographic structures: numerical comparison with second gradient continuum models. Zeitschrift für angewandte Mathematik und Physik, ZAMP, 67(85), pp. 01-28, 2016

[16] Abali, B.E., Müller, W.H., Eremeyev, V.A. Strain gradient elasticity with geometric nonlinearities and its computational evaluation Mechanics of Advanced Materials and Modern Processes, 1(1), pp. 01-11, 2015

[17] Pau, A., Trovalusci, P. Block masonry as equivalent micropolar continua: The role of relative rotations. Acta Mechanica, 223(7), pp. 1455-1471, 2012

[18] Jing, G.Y., Duan, H.L., Sun, X.M., Zhang, Z.S., Xu, J., Li, Y.D., Wang, J.X., Yu, D.P. Surface effects on elastic properties of silver nanowires: Contact atomicforce microscopy. Physical Review B, 73, pp. 01-06, 2006 